\title{
Cempestón
}

\section{Personal information management e seu impacto na produção científica de pesquisadores do domínio da educação}

\author{
Helen de Castro S. Casarin \\ Doutora; Universidade Estadual Paulista Júlio de Mesquita Filho, São Paulo, SP, Brasil; \\ helen.castro@unesp.br; ORCID: https://orcid.org/0000-0002-3997-9207 \\ Cátia Candida Almeida \\ Doutora; Fundação Educacional de Penápolis, Penápolis, SP, Brasil; \\ catiacandida@yahoo.com.br; ORCID: https://orcid.org/0000-0002-8477-6257
}

Resumo: O desenvolvimento de tecnologias de informação tem afetado a forma como pesquisadores lidam com a informação na produção do conhecimento. Realizou-se um estudo com o objetivo de identificar as estratégias e os recursos utilizados por pesquisadores brasileiros do domínio Educação para organizar suas coleções; verificar se as dificuldades no manejo destas coleções afetam a produção científica destes pesquisadores e investigar a influência de variáveis demográficas nestas atividades. Para a coleta de dados foi aplicado um questionário eletrônico a pesquisadores vinculados às dez universidades brasileiras melhor colocadas no ranking da Folha de São Paulo em três avaliações subsequentes. Obteve-se 205 respostas, porém apenas 163 questionários foram considerados válidos pelo fato de estarem completos ou parcialmente respondidos. Os demais continham apenas os dados demográficos dos respondentes ou estavam em branco. Os dados coletados foram analisados quantitativamente utilizando-se o teste estatístico qui-quadrado para verificar a associação da distribuição de respostas dos participantes entre as variáveis do estudo. Os resultados demonstraram que a minoria dos participantes utiliza ferramentas específicas para gerenciar suas coleções. Verificou-se, ainda, que a dificuldade em gerenciar suas coleções tem impactado na produção científica dos pesquisadores e que aqueles que passaram por algum treinamento sobre ferramentas para organizar e armazenar seus documentos enfrentam menos dificuldades para recuperar materiais de sua coleção pessoal. Pretende-se que os resultados da pesquisa possam servir de base para o preparo de bibliotecários para o atendimento a este grupo de pesquisadores e para a adaptação e proposição de produtos e serviços informacionais relacionados ao Personal Informantion Management.

Palavras-chave: Comportamento de busca; Coleções pessoais; Produção científica; Organização da informação 


\section{Introdução}

A produção do conhecimento, que é própria do ofício do pesquisador, requer a constante busca de informações científicas e a manutenção de coleções pessoais de documentos científicos de natureza e formatos variados. Como lembram Athukorala et al. (2013) e Al-Omar e Cox (2013), a busca de informações relevantes, a verificação da qualidade de informações e o uso de informações no processo de realização das pesquisas fazem parte do cotidiano e são essenciais aos pesquisadores. No entanto, o desenvolvimento de tecnologias, em particular da internet, tem provocado mudanças nestas práticas. Conforme Warraich, Ali e Yasmeen (2018), fatores como a explosão informacional, os avanços tecnológicos e o uso cada vez mais intenso de tecnologia digital no processo de ensino-aprendizagem tornam o trabalho de organização e guarda de informações acadêmicas pelos docentes mais desafiador do que nunca.

Estudos têm demonstrado que o manejo adequado de informações acadêmicas pelos pesquisadores pode minimizar o estresse, economizar tempo, aumentar a eficiência e, até mesmo, diminuir a sensação de sobrecarga de informações (ETZEL; THOMAS, 1996), além de contribuir para a melhoria da qualidade das atividades acadêmicas (DIEKEMA; OLSEN, 2014 apud $^{1}$ WARRAICH; ALI; YASMEEN, 2018).

O foco do presente artigo é investigar como os pesquisadores do domínio da Educação têm lidado com suas coleções de documentos científicos, incluindo suas práticas e o impacto da manutenção destas coleções nas suas produções científicas. Os objetivos do estudo foram: identificar as estratégias e os recursos utilizados por pesquisadores brasileiros do domínio Educação para organizar suas coleções; verificar se as dificuldades no manejo destas coleções afetam a produção científica destes pesquisadores e investigar a influência de variáveis demográficas como idade e gênero, entre outras, nestas atividades.

\section{Comportamento informacional e o Personal Information Management - PIM}

$\mathrm{O}$ ato de buscar, organizar e usar informação é um comportamento muito comum e essencial aos seres humanos (CASE; GIVEN, 2016). Para Wilson (2000), o comportamento informacional abrange todo o comportamento humano 
relacionado às fontes e canais de informação. O comportamento informacional inclui desde a busca passiva, em que os indivíduos se deparam com informações relevantes, mesmo não realizando uma busca formal, até a busca ativa, na qual há a intenção de se obter uma informação específica. Compreende também a obtenção, o armazenamento, a apropriação e o uso da informação. Inclui, ainda, as ações para evitar determinadas informações, por exemplo, quando se trata de um assunto que causa desconforto.

Os estudos sobre comportamento informacional muitas vezes abrangem o processo de busca de informação pelos indivíduos ou partes deste processo. No entanto, o uso que se faz das informações que são obtidas é pouco explorado nas pesquisas da área, conforme ressaltam Case e Given (2016). Case e O’Connor (2016) levantam uma série de autores que apontam para a ambiguidade do conceito de "uso da informação" e dos termos que muitas vezes são usados como sinônimos. Para Kari (2007 apud ${ }^{2}$ CASE; O’CONNOR, 2016), o termo outcomes abrange tanto o que o sujeito faz com a informação obtida, ou seja, sua aplicação, como os efeitos causados ou decorrentes da informação obtida. No caso desta pesquisa, foi adotado o primeiro significado.

Koh et al. (2015) relatam a discussão de especialistas em um painel sobre a importância de se investigar o comportamento informacional relacionado à organização de informações no dia a dia, em particular o processo adotado pelas pessoas para realizar tal atividade. Segundo os autores, nas discussões do painel foi apontado que a forma como as pessoas manejam a informação para fins pessoais ou de trabalho é um componente do comportamento informacional que vem sendo pouco investigado. Os resultados das pesquisas sobre este tema podem gerar subsídios para elaboração de sistemas e ferramentas que sirvam de suporte ao trabalho de manejo de informações em diferentes contextos.

Ações envolvidas no armazenamento da informação para uso futuro são denominadas como Personal Information Management (PIM). Este termo, no entanto, foi usado pela primeira vez na década de 1980 ante a expectativa sobre o potencial do computador pessoal para ampliar a habilidade das pessoas em lidarem com a informação (JONES, 2007, p. 458). PIM pode ser definido como: 
[...] a prática e o estudo das atividades que uma pessoa realiza a fim de adquirir ou criar, armazenar, organizar, manter, recuperar, usar e distribuir as informações necessárias para completar tarefas e cumprir várias funções e responsabilidades. (JONES, 2007, p. 453, tradução nossa).

Mais recentemente, o PIM ou Information-keeping behavior foi definido por Koh et al. (2015, tradução nossa) como "[...] a forma como as pessoas mantêm as informações em um local físico ou virtual por um determinado período de tempo para uso pessoal ou organizacional.”.

Os estudos sobre a temática podem abranger vários aspectos sobre como as pessoas lidam com as informações que elas mantêm em suas coleções pessoais (JONES, 2007). Conforme Paré (2011), os primeiros estudos relacionados a esse assunto estavam inseridos, embrionariamente, em pesquisas sobre comportamento informacional. Para o autor, embora esses estudos estivessem mais focados na quantidade de documentos presentes em um escritório, por exemplo, a organização e o uso destes documentos também faziam parte dos interesses destas pesquisas e, com o passar do tempo, passaram a se constituir em uma nova linha de pesquisa (PARÉ, 2011). Então, antes mesmo que houvesse alguma menção ao termo Personal Information Management como tema de pesquisa propriamente dito, ele era visto como parte dos estudos de comportamento informacional (PARÉ, 2011).

Os estudos sobre comportamento informacional continuam a influenciar a formação e aplicação dos estudos sobre PIM. Conforme Greifeneder (2014), embora a temática da busca ativa por informação seja predominante nas pesquisas sobre comportamento informacional, nos últimos anos outros temas como a criação/produção da informação, a guarda (saving) e a aprendizagem têm se fortalecido. A renovação do interesse sobre a PIM apresenta-se possivelmente em resposta à conscientização da problemática que as novas tecnologias podem criar nos ambientes informacionais (JONES; TEEVAN, 2007), pois com o desenvolvimento das ferramentas tecnológicas houve a necessidade de reformulação de estratégias para organização de coleções pessoais adequando-as a este novo contexto, principalmente no último ano por conta do trabalho remoto. 


\subsection{Personal Information Management (PIM)}

Os sistemas de Personal Information Management (PIM) são mais reservados e de acesso restrito, pois são desenvolvidos e/ou criados para o uso pessoal de um indivíduo em seu ambiente de trabalho (BARREAU; NARDI, 1995), que pode ser inclusive em sua própria casa.

O PIM envolve algumas atividades intrinsecamente relacionadas e que são necessárias ao gerenciamento de coleções pessoais, quais sejam: encontrar e reencontrar; guarda e organização da informação. Essas atividades são descritas por Jones (2007) em termos de necessidade e informação. De maneira geral, indivíduos encontram ou reencontram informação com vistas a satisfazer uma necessidade de informação. Para tanto, eles se engajam em um processo de busca para obtê-las. Pode ocorrer também uma busca não estruturada em que os sujeitos se deparam com informações potencialmente úteis ainda que não estejam realizando uma busca específica ou estejam procurando algo bastante diferente do que foi encontrado, denominada seredipity (CASE; GIVEN, 2016; VECHIATO; FARIAS, 2020). Após encontrar as informações avaliadas como úteis, elas podem ser usadas ou mantidas (armazenadas) com o intuito de suprir necessidades informacionais futuras.

As atividades de gerenciamento e organização da informação implicam em decidir quando, onde e de qual maneira as informações serão mantidas para que a sua localização futura seja, de fato, possível. Essas últimas atividades vão permitir que a informação armazenada seja localizada posteriormente. Porém, em geral, essas tarefas são deixadas para um momento subsequente por não serem consideradas urgentes.

Segundo Oh (2013), as atividades de PIM podem ser agrupadas em três tipos principais: atividades de guarda, atividades de meta-nível (organização e manutenção) e atividades de encontro e reencontro. A atividade de guarda é considerada como pré-requisito para as demais, pois para que o item informacional seja organizado e mantido depende de ter sido previamente selecionado e guardado. Além disto, quanto mais itens são adicionados à coleção do pesquisador, mais difícil e complexo se tornam as atividades de 
organização e encontro/reencontro ou recuperação. As atividades de meta-nível podem facilitar ou dificultar a terceira atividade, que diz respeito às ações de reencontrar e encontrar Isto se deve ao fato de que a forma como os itens informacionais são organizados na coleção do pesquisador pode facilitar ou dificultar a sua recuperação, podendo até mesmo inviabilizá-la, caso a organização tenha sido feita de maneira inadequada. A atividade encontrar/reencontrar informações, por sua vez, indica como os itens serão mantidos e utilizados no futuro, mostrando assim como as atividades influenciam umas às outras dentro da prática do Personal Information Management.

Feitas as considerações mais gerais sobre PIM, serão apresentados a seguir os estudos sobre as práticas de pesquisadores na área de humanidades relacionadas ao tema.

\section{Manejo de coleções pessoais por pesquisadores do domínio da Educação}

A relação entre eficiência dos pesquisadores e organização de suas coleções pessoais há muito vem sendo pensada. O artigo clássico de V. Bush (1945), por exemplo, apontava a necessidade de aumentar a eficiência de pesquisadores através de um sistema de armazenamento e organização de documentos variados de suas coleções pessoais.

Outro fator a ser considerado é que o pesquisador faz uso de informação especializada que, anteriormente à expansão da Web, estava disponível principalmente em bibliotecas vinculadas a institutos de pesquisa e de ensino superior, agora se vê embebido pelas ferramentas de busca [e manejo de informações] eletrônicas (HEMMINGER et al., 2007) sem a necessidade de uma mediação direta do bibliotecário. Embora haja a mediação indireta através da organização da informação em catálogos, bases de dados e outros instrumentos e recursos informacionais, o pesquisador lida em seu cotidiano com tarefas relacionadas ao manejo de informações disponíveis em um volume cada vez maior e, em boa medida, fora dos muros reais ou virtuais da biblioteca.

Se esta dificuldade de organização das coleções pessoais já existia no contexto em que o suporte impresso era predominante, com a disponibilização 
de informações de forma eletrônica ela se potencializou. McGeachin (2004) descreve a passagem da coleção pessoal predominantemente impressa para coleções com itens digitais obtidos através de bases de dados e internet. Neste período, no início dos anos 2000, muitos pesquisadores optavam por passar o impresso para o digital através de scanners para que pudesse agrupá-los à crescente coleção eletrônica. Havia aqueles que faziam o inverso, imprimindo artigos que lhes eram relevantes, agregando-os às suas coleções de fotocópias e pré-prints.

Newman e Sack (2013) confirmam o impacto causado pelas mudanças tecnológicas nas práticas de pesquisa. Segundo os autores, a transição do formato impresso de livros e periódicos para o formato digital modificou o trabalho e os métodos usados pelos pesquisadores para encontrar, armazenar, recuperar e usar informações.

Apesar da aparente facilidade para guarda destes materiais proporcionada pela tecnologia, paradoxalmente é difícil reencontrar um material específico. Isso é causado, por exemplo, pela dificuldade e diversidade de tipos de dados, opções para armazenamento e pela fragmentação do material. Além disso, em alguns casos, a informação e seus registros são obtidos para uso futuro. Assim, os indivíduos precisam ter habilidades de PIM para conseguirem armazenar e organizar as informações eficientemente para que possam retornar a elas futuramente quando forem necessárias (STEWART; BASIC, 2014).

O impacto da proliferação de recursos eletrônicos para o manejo de informações científicas visando a produção do conhecimento, que é o interesse da pesquisa aqui relatada, tem sido apontado por diversos autores há alguns anos. Entre os autores que têm se destacado no estudo desta temática estão: William Jones (JONES, 2007; JONES; TEEVAN; JONES, 2007; JONES; WENNING; BRUCE, 2014; JONES et al., 2016), Carol Tenopir (TENOPIR, 2003; TENOPIR et al. 2015), Donald W. King (KING; TENOPIR, 2001) e Bradley M. Hemminger (2007), entre outros.

Fry (2006) foi um dos primeiros autores a investigar o impacto das tecnologias de informação e comunicação em práticas de pesquisa. O autor utilizou a abordagem de análise de domínio e verificou que o local de trabalho e 
as práticas de comunicação de pesquisadores em diferentes campos do conhecimento influenciam o uso de recursos digitais em comunicações formais e informais. $\mathrm{O}$ autor demonstrou também que práticas informais de comunicação moldam a produção e o uso de infraestrutura de informação digital.

Hemminger et al. (2007) chamam a atenção para as mudanças provocadas pela proliferação da quantidade e variedade de recursos informacionais mesmo em áreas específicas como ciência e medicina, que inclui desde as fontes tradicionais como livros, periódicos científicos, relatórios, bases de dados de científicas, e outras não convencionais como webpages, listas de discussão, wikis, e as mídias sociais que têm sido utilizadas para busca e divulgação de informações científicas (TENOPIR et al., 2015). Hemminger et al. (2007) também apontam que os próprios mecanismos tradicionais para busca, recuperação e visualização de conteúdos estão mudando dramaticamente.

Estudos mais recentes (NIU; HEMMINGER, 2012; WELLINGS; CASSELDEN, 2017; HASSAN et al., 2017; ARSHAD; AMEEN, 2021), demonstram que os recursos eletrônicos baseados em web são a principal fonte de informação científica para a maior parte dos pesquisadores de diferentes áreas científicas. A presença constante e cada vez mais intensa de buscadores, especificamente o Google, entre os cientistas vem sendo apontada em pesquisas de forma recorrente (JAMALI; ASADI (2010), KEMMAN; KLEPPE; SCAGLIOLA (2012), GIVEN; WILLSON (2018) entre outros).

Com a disponibilidade de tantos recursos e informações para serem manejadas, há a necessidade de se desenvolver estratégias para organizar e recuperar as informações encontradas e armazenadas em coleções pessoais. Ou, então, como dizem Bruce, Jones e Dumais (2004), as pessoas manejam informações para acesso futuro e reuso, que inclui atividades de guarda das informações consideradas como úteis ou relevantes, ou deixando-as onde foram encontradas ao invés de incorporá-las à coleção pessoal neste caso o indivíduo se considera capaz de recuperá-las posteriormente, reencontrando-as ("keepingleaving and re-finding”).

A pesquisa de Jones, Wenning e Bruce (2014) identificou o esforço e o tempo gasto por pesquisadores para manter sua coleção de documentos, que 
inclui e-mails, arquivos eletrônicos e webpages organizadas de forma a possibilitar sua recuperação posterior. A pesquisa de Henderson (2009 apud ${ }^{3}$ AL-OMAR; COX, 2013) demonstrara que, embora as pessoas considerem a manutenção de coleções pessoais como algo importante, elas tentam gastar o mínimo de tempo nesta tarefa, o que tem consequências na recuperação posterior destes documentos.

Conforme Hwang, Lin e Shin (2018) e Warraich, Ali e Yasmeen (2018), um melhor domínio do PIM pode resultar em uma otimização do uso de preciosos e escassos recursos (tempo, energia e atenção) e melhorar o resultado das atividades acadêmicas contudo, que nem todos apresentam a mesma performance nesta tarefa, justamente devido a fatores como motivação e capacidade, entre outros fatores.

AL-Anazi, Hinze e Vanderschantz (2014) realizaram um estudo para verificar como pesquisadores lidavam com suas coleções em suporte eletrônico. Em geral, os participantes do estudo armazenavam os artigos e outros documentos para leitura posterior em pastas em seu próprio desktop. Eles apontaram os seguintes problemas decorrentes desta prática: “[...] passar muito tempo procurando informações ou um determinado documento, ler um artigo mais de uma vez e o fato de ter que duplicar arquivos com diferentes destaques e comentários." (AL-ANAZI, 2013, p.28, tradução nossa). Bergman e Yanai, (2018) verificaram a tendência de uso de novos suportes, como smartphones, além dos computadores para armazenamento de itens informacionais por pesquisadores.

O estudo de Al-Omar e Cox (2013) indicou que os pesquisadores desenvolvem suas coleções ao longo da realização de seus projetos. O uso destes materiais é recorrente, ou seja, está presente em várias fases de realização da pesquisa. Além disto, o material coletado ao longo das pesquisas, em geral, não é descartado, mas guardado e reutilizado em outros projetos. Estes resultados demonstram a importância de um gerenciamento otimizado das coleções pessoais.

Oh (2013) também ressalta que a forma como os sujeitos manejam a informação varia entre grupos sociais ou comunidades de pensamento. Tal 
postura se alinha à abordagem de análise de domínio, a qual, a nosso ver, é bastante adequada aos estudos de comportamento informacional e foi adotada neste estudo (HJØRLAND, 2017).

A área de Educação, que é uma confluência de conhecimentos, práticas e políticas de origens diversas (CHARLOT, 2006), contém características do domínio de alta dispersão (high scatter domains) proposto por Mote (1962 apud $^{4}$ TALJA; MAULA, 2003; BATES, 1996). Segundo Bates (1996, p. 156, tradução nossa)

Em domínios de alta dispersão, a cobertura de assuntos é ampla [dado o grande número de temas de pesquisa investigados] e a organização literatura não é clara ou é pouco útil considerando os interesses e problemas de pesquisa da área ${ }^{5}$.

Bates (1996) salienta, ainda, que a busca de informações em campos interdisciplinares requer que o pesquisador consulte várias disciplinas para localizar informações relevantes.

Assim, o propósito da pesquisa aqui relatada foi verificar em que medida a disponibilidade de recursos eletrônicos para manipulação (busca, recuperação, armazenamento, uso e compartilhamento) de informações científicas tem influenciado o comportamento informacional de pesquisadores brasileiros no domínio da Educação.

Neste artigo serão relatadas as dificuldades encontradas pelos pesquisadores no manejo de informações científicas para produção do conhecimento e a influência de variáveis demográficas como idade e gênero, no manejo das informações científicas.

\section{Delineamento da pesquisa}

O universo da pesquisa foi composto por docentes de Programas de PósGraduação em Educação das universidades que se mantiveram entre as 10 primeiras posições, segundo o ranking da Folha de São Paulo, em três avaliações subsequentes antes da realização da coleta de dados (2013, 2014, 2016), a saber: Universidade de São Paulo, Universidade Estadual de Campinas, Universidade Federal do Rio de Janeiro, Universidade Federal de Brasília, Universidade Estadual Paulista, Universidade Federal de Minas Gerais e 
Universidade Federal do Rio Grande do Sul, Universidade Federal de Pernambuco, Pontifícia Universidade Católica de Campinas e Universidade Federal Fluminense.

Considerando que a taxa de retorno estimada é de $30 \%$ a $40 \%$, optou-se por enviar o questionário a todos os docentes dos referidos programas. Para tanto, foram consultadas as páginas dos cursos de Pós-graduação em Educação e coletados os e-mails disponíveis, totalizando 793 docentes. Em seguida os endereços de e-mails foram incluídos na plataforma SurveyMonkey e o questionário foi enviado diretamente aos docentes.

A coleta de dados foi realizada entre novembro de 2017 e março de 2018. Vinte questionários retornaram, provavelmente pelo fato do e-mail do docente estar incorreto ou por este fazer uso de AntiSpam. Sete respondentes cancelaram o envio do questionário recusando-se participar da pesquisa. Assim, foram obtidas 205 respostas (25,8\% do total de possíveis respondentes), sendo que 182 concordaram em participar da pesquisa e 23 assinalaram a opção "não participar" incluída na página inicial do questionário. A taxa de preenchimento completo do questionário, dentre os participantes, foi de 153 questionários e 28 de forma parcial. 163 (20,5\% do total de possíveis respondentes) questionários foram considerados válidos para análise, pois estavam preenchidos completamente ou com apenas poucas questões sem preencher.

O questionário utilizado na coleta de dados era composto por 15 questões, incluindo cinco questões de caracterização e dez questões relacionadas ao comportamento dos participantes quanto ao gerenciamento de suas coleções pessoais de materiais acadêmicos. O questionário era acompanhado do Termo de Consentimento Livre e Esclarecido.

Quanto ao perfil dos 163 respondentes, verificou-se que $110(67,48 \%)$ eram do sexo feminino e $53(32,52 \%)$ do sexo masculino. Quanto à idade, verificou-se que a maior parte dos participantes concentra-se nas faixas entre 41 e 60 anos, como demonstra a Tabela 1, sendo, em tese, pesquisadores experientes. 
A maioria dos respondentes era de instituições públicas $(99,39 \%)$ e apenas um (01) de instituição privada. 93,25\% dos participantes tinham tempo de trabalho acima de seis anos.

Observou-se a relação entre idade e vínculo dos participantes com os tipos de programas de pós-graduação, conforme demonstra a Tabela 1. Destacase que $135(82,8 \%)$ participantes apresentaram vínculo com pós-graduação Stricto Senso.

Tabela 1 - Idade dos participantes e vínculo dos participantes a programas de pós-graduação

\begin{tabular}{cccccc}
\hline Idade & $\begin{array}{c}\text { Sim, stricto } \\
\text { senso }\end{array}$ & $\begin{array}{c}\text { Sim, lato } \\
\text { senso }\end{array}$ & $\begin{array}{c}\text { Não estou } \\
\text { vinculado }\end{array}$ & Total & $\%$ \\
\hline Até 40 anos & 19 & 5 & 8 & 32 & 19,6 \\
$41-50$ anos & 42 & 2 & 4 & 48 & 29,4 \\
$51-60$ anos & 46 & 5 & 3 & 54 & 33,1 \\
61-70 anos & 22 & 0 & 1 & 23 & 14,1 \\
Acima de 70 & 6 & 0 & 0 & 6 & 3,7 \\
anos & 135 & 12 & 16 & 163 & 100 \\
Total & Fonte: Elaborado pelos autores (valor de $\mathrm{p}=0,015)$. &
\end{tabular}

Os dados foram analisados utilizando o software Statistical Package for the Social Sciences (SPSS) versão 24. Aplicou-se o teste estatístico QuiQuadrado com propósito de verificar a associação da distribuição de respostas dos participantes entre as variáveis do estudo, considerando o nível de significância de $5 \%$.

\section{Resultados}

Os participantes foram questionados se sentiam dificuldades para recuperar itens de sua coleção pessoal. A maioria dos participantes respondeu afirmativamente, ainda que esta dificuldade não seja tão frequente, como demonstra a Tabela 2, visto que as respostas se concentraram nas categorias "algumas vezes" ou "raramente".

Tais resultados coincidem com a pesquisa de Al-Anazi (2013) que apontou para a dificuldade dos pesquisadores em recuperar itens de suas coleções pessoais.

Tabela 2 - Dificuldade dos sujeitos em recuperar itens da coleção pessoal dos participantes

\begin{tabular}{lcc} 
Resposta & Frequência & $\%$ \\
\hline Sim, sempre & 1 & 0,6 \\
\hline
\end{tabular}




\begin{tabular}{lcc}
\hline Sim, frequentemente & 8 & 4,9 \\
Sim, algumas vezes & 56 & 34,4 \\
Sim, raramente & 39 & 23,9 \\
Não se aplica ao meu caso & 59 & 36,2 \\
Total & 163 & 100,0 \\
\hline
\end{tabular}

Fonte: Elaborado pelos autores.

Destaca-se que $34,4 \%$ e $23,9 \%$ dos participantes responderam respectivamente "Sim, algumas vezes" e "Sim, raramente". Este fato demonstra que os participantes têm algumas dificuldades na recuperação das informações da coleção pessoal e que tal fato pode ter um impacto significativo na produção acadêmica, mesmo entre pesquisadores das melhores universidades brasileiras. Procurou-se averiguar a relação entre as dificuldades dos participantes para reencontrar/recuperar os documentos de sua coleção pessoal considerando sexo ou idade, mas não foi identificada uma associação significativa entre as variáveis.

Tabela 3 - Impacto das dificuldades em recuperar informações de coleção pessoal na produção acadêmica dos participantes

\begin{tabular}{|c|c|c|}
\hline Resposta & Frequência & $\%$ \\
\hline Sim, sempre & 1 & 0,6 \\
\hline Sim, frequentemente & 9 & 5,5 \\
\hline Sim, algumas vezes & 58 & 35,6 \\
\hline Sim, raramente & 40 & 24,5 \\
\hline $\begin{array}{l}\text { Não tenho } \\
\text { dificuldades }\end{array}$ & 55 & 33,7 \\
\hline Total & 163 & 100,0 \\
\hline
\end{tabular}

Fonte: Elaborado pelos autores.

Verificou-se se as dificuldades em recuperar informações da coleção pessoal têm impacto na produção acadêmica dos participantes (Tabela 3). Notase que a maioria dos respondentes considera que as dificuldades em recuperar itens de coleção pessoal têm impactado na produção acadêmica, ainda que a frequência seja dividida entre "algumas vezes" (35,6\%) e "raramente" $(24,5 \%)$. Por outro lado, 33,7\% participantes afirmaram não sentir dificuldades. Procurou-se averiguar a relação entre dificuldade em recuperar itens de sua coleção pessoal e as variáveis sexo e idade dos participantes, porém o teste estatístico não revelou associação significativa entre estas variáveis. 
Considerando que a dificuldade em recuperar materiais de suas coleções pode, inclusive, afetar negativamente a produtividade dos pesquisadores, verificou se eles haviam realizado treinamento para uso de ferramentas para organizar e armazenar documentos de sua coleção pessoal (Gráfico 1).

Gráfico 1: Distribuição de frequência do interesse e participação dos sujeitos em treinamentos sobre ferramentas de PIM

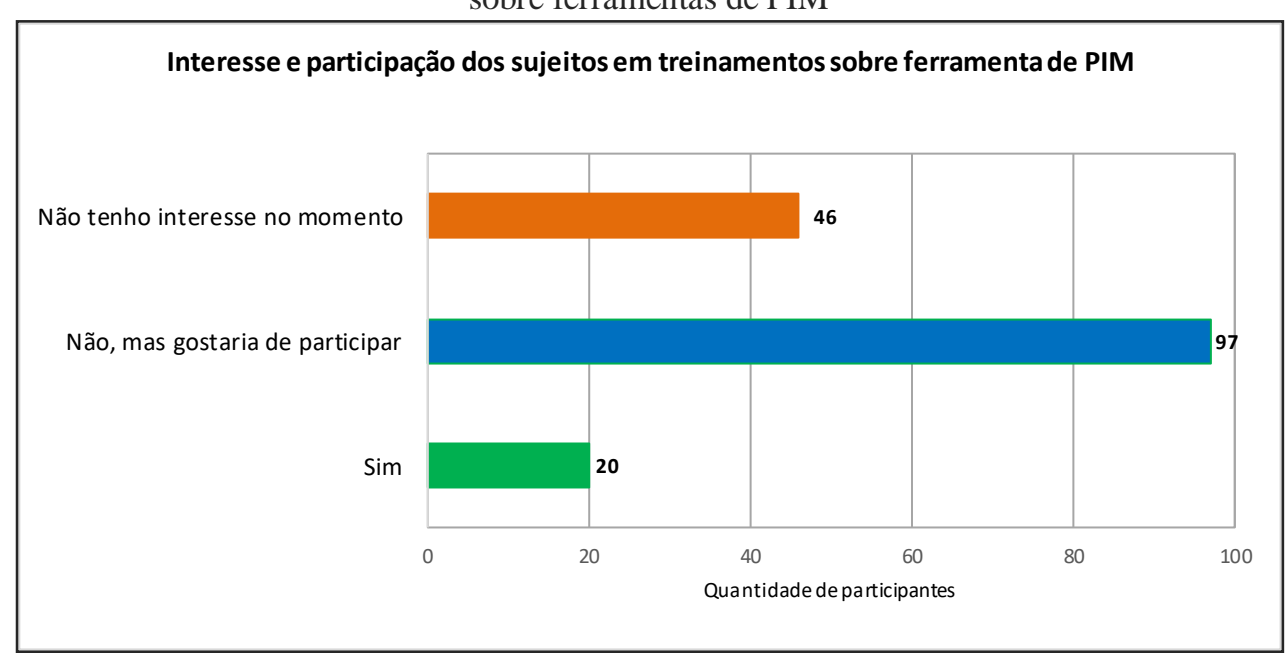

Fonte: Elaborado pelos autores.

Conforme o Gráfico 1, apenas 20 (12,3\%) participantes responderam afirmativamente, o que é um valor muito baixo considerando que ferramentas importantes poderiam facilitar ou agilizar o trabalho dos pesquisadores, ainda mais considerando que o universo da pesquisa é constituído por docentes das melhores universidades brasileiras.

A maior parte dos respondentes, no entanto, apesar de não ter participado de treinamento, 55,4\% (97), demonstrou interesse em participar, o que sinaliza que as bibliotecas das instituições poderiam propor este tipo de treinamento, já que há demanda para tal.

Conforme o Gráfico 1, um percentual considerável 28,2\% (46) dos participantes, no entanto, afirmou não ter interesse em participar. Este percentual é próximo ao de respondentes que afirmaram não sentir dificuldades $(33,7 \%)$, conforme o indicado na Tabela 3 , o que será melhor explorado mais a frente neste artigo. Deste modo, é possível que, por não sentirem esta dificuldade, eles não tenham interesse em participar dos treinamentos. Pode ser ainda que haja necessidade de uma conscientização ou, ao menos, um 
esclarecimento dos benefícios de uso destas ferramentas para gerenciamento de coleções pessoais aos pesquisadores.

Constatou-se uma associação estatística significativa entre as variáveis participação em treinamento sobre ferramentas de PIM e sexo, indicando uma tendência dos sujeitos de sexo feminino, que ainda não participaram de treinamento, terem mais pretensão em participar que os sujeitos do sexo masculino.

Baseado na afirmação de $55(33,7 \%)$ participantes de que não sentem dificuldades em recuperar informações da coleção pessoal, optou-se por investigar com mais detalhes a relação daqueles que sentem algum tipo de dificuldade e outras variáreis. Assim, os participantes do estudo foram divididos em dois grupos: aqueles que sentem algum grau de dificuldade (108 participantes) e aqueles que não sentem dificuldades (55 participantes). Os resultados estão reunidos nas tabelas a seguir.

$\mathrm{Na}$ Tabela 4 verificou-se a relação entre grau de dificuldade em recuperar documentos da coleção pessoal e o interesse dos sujeitos em participar de treinamento sobre ferramentas de PIM. A Tabela 4 aponta que grande parte dos que sentem dificuldades, embora não tenham participado de treinamento, demonstram interesse em participar (72 participantes). 24 afirmaram que sentem algum grau de dificuldade, mas não têm interesse em participar e 12, mesmo tendo participado, ainda sentem dificuldades, demonstrando a necessidade de um auxílio ou complementação da formação.

Tabela 4 - Grau de dificuldade dos participantes em recuperar documentos e participação em treinamentos sobre ferramentas de PIM

\begin{tabular}{lccccc}
\hline $\begin{array}{l}\text { Grau de dificuldade } \\
\text { participantes }\end{array}$ & sentida pelos & Sim & $\begin{array}{c}\text { Não, mas } \\
\text { tenho interesse }\end{array}$ & $\begin{array}{c}\text { Não tenho } \\
\text { Interesse }\end{array}$ & Total \\
\hline Sentem algum grau de dificuldade & 12 & 72 & 24 & 108 \\
Não sentem dificuldade & 8 & 25 & 22 & 55 \\
Total & 20 & 97 & 46 & 163 \\
\hline
\end{tabular}

Fonte: Elaborado pelos autores (valor de $\mathrm{p}=0,028$ ).

Entre os que não sentem dificuldade (Tabela 4), apenas oito já participaram de treinamento. 25 participantes, mesmo não sentido dificuldades, têm interesse em participar e 22 participantes do grupo que "não sente 
dificuldade" ao recuperar documentos de sua coleção pessoal, não têm interesse de participar de treinamento sobre ferramentas de PIM. O teste estatístico indicou que existe relação entre os grupos dos que "sentem alguma dificuldade" e os que "não sentem difículdade" em recuperar os documentos de suas coleções pessoais e o interesse de participar de treinamentos sobre ferramentas de PIM, demonstrando que a percepção da dificuldade é um fator que predispõe o participante a ter mais interesse em participar de treinamentos sobre a temática em questão.

Verificou-se ainda a relação entre a frequência com que ocorrem as dificuldades em recuperar documentos pelos respondentes e a participação deles em treinamentos sobre ferramentas PIM (Tabela 5).

Tabela 5 - Participação em treinamento e frequência com que sentem dificuldade em recuperar documentos pessoais

\begin{tabular}{lccccc}
\hline $\begin{array}{l}\text { Frequência de dificuldades } \\
\text { recuperar documentos }\end{array}$ & em & Sim & $\begin{array}{c}\text { Não, mas } \\
\text { tenho interesse }\end{array}$ & $\begin{array}{c}\text { Não tenho } \\
\text { Interesse }\end{array}$ & total \\
\hline Sempre & 0 & 1 & 0 & 1 \\
Frequentemente & 0 & 8 & 0 & 8 \\
Algumas vezes & 7 & 40 & 9 & 56 \\
Raramente & 7 & 19 & 13 & 39 \\
Não tenho sentido dificuldades & 6 & 29 & 24 & 59 \\
Total & 20 & 97 & 46 & 163 \\
\hline
\end{tabular}

Fonte: Elaborado pelos autores (valor de $\mathrm{p}=0,029$ ).

Entre as 163 respostas, destacam-se aqueles que indicaram que sentem dificuldades "algumas vezes" e ao mesmo tempo não têm interessem em participar de treinamento com 40 assinalações e também aqueles que afirmaram que não tinham sentido dificuldades e que afirmaram não terem participado de treinamento, mas que têm interesse em fazê-lo, com 29 assinalações. O teste estatístico apontou diferenças significativas na distribuição de respostas desses participantes, a saber, entre o fato de os sujeitos terem passado por treinamento sobre as ferramentas de PIM e a dificuldade de recuperar documentos em suas coleções pessoais.

Este fato pode evidenciar que quem já passou por algum treinamento sobre ferramentas para organizar e armazenar seus documentos apresenta menos dificuldades para recuperar materiais de sua coleção pessoal, o que reforça a 
importância de se oferecer treinamentos e trabalhar na conscientização dos docentes do domínio de Educação a respeito dos mesmos.

\section{Considerações finais}

O propósito do estudo foi identificar se os pesquisadores brasileiros do domínio Educação encontram dificuldades no manejo de informações científicas para produção do conhecimento e investigar a influência de variáveis demográficas como idade e gênero, entre outras, nestas atividades. O estudo demonstrou que os pesquisadores das melhores universidades do país no quesito pesquisa têm sentido dificuldades em relação ao aspecto estudado na pesquisa.

Os resultados demonstram também que as dificuldades sentidas pelos docentes têm impactado, ainda que com uma baixa frequência, a sua produção acadêmica. Considerando que esta é uma atividade pela qual os pesquisadores são bastante cobrados, tendo muitas vezes que cumprir metas de publicação impostas pelos programas de pós-graduação, agências regulatórias e de fomento, ter algo que atrapalhe a sua realização é bastante significativo.

$\mathrm{O}$ fato de que apenas um pequeno percentual dos pesquisadores $(12,3 \%)$ afirmou já haver participado de treinamento sobre as ferramentas de PIM revela a necessidade premente de uma ação das bibliotecas e das universidades no sentido de instrumentalizar seus pesquisadores, em particular os do domínio da Educação. Percebe-se que, de maneira geral, mesmo entre as melhores universidades brasileiras, ainda não há uma cultura de formação e atualização dos pesquisadores em relação às ferramentas PIM.

As dificuldades dos pesquisadores relacionadas ao manejo de suas coleções e o desinteresse em se atualizar para o uso de ferramentas PIM pode ter reflexo também na formação de outros pesquisadores, que são orientados por eles, perpetuando esta prática. Cabe às universidades e às bibliotecas universitárias despertar o interesse da comunidade acadêmica para estas questões e buscar estratégias para propiciar a formação de seus membros.

Espera-se que estes resultados possam servir de base para o preparo de bibliotecários e para a proposição de produtos e serviços informacionais voltados para esta área. 


\section{Referências}

AL-ANAZI, M.; HINZE, A.; VANDERSCHANTZ, N. Personal digital libraries: Keeping track of academic reading material. In: INTERNATIONAL CONFERENCE ON ASIAN DGITAL LIBRARIES, [2014?], Cham. Proceedings [...]. Cham: Springer, 2014. p. 39-47.

AL-ANAZI, M. S. Keeping track of electronic reading material. Tese (Doutorado em Ciência da Computação) - University of Waikato, Hamilton, 2013.

AL-OMAR, M.; COX, A. Finders, keepers, losers, seekers: a study of academics' research-related personal information collections. In: INTERNATIONAL CONFERENCE ON HUMAN INTERFACE AND THE MANAGEMENT OF INFORMATION, [2013?], Berlin. Proceedings [...]. Berlin, Heidelberg: Springer, 2013. p. 169-176.

ARSHAD, A.; AMEEN, K. Comparative analysis of academic scientists, social scientists and humanists' scholarly information seeking habits. The Journal of academic librarianship, New York, v. 47, n. 1, p. 1-9, 2021.

ATHUKORALA, K. et al. Information-seeking behaviors of computer scientists: Challenges for electronic literature search tools. Proceedings of the American Society for Information Science and Technology, [S.l.], v. 50, n. 1, p. 1-11, 2013.

BARREAU, D.; NARDI, B. A. Finding and reminding: file organization from the desktop. ACM Sigchi Bulletin, [S.l.], v. 27, n. 3, p. 39-43, Jul. 1995.

BATES, M. J. Learning about the information seeking of interdisciplinary scholars and students. Library trends, Baltmore, v. 45, n. 2, p. 155-64, 1996.

BERGMAN, O.; YANAI, N. Personal information retrieval: smartphones vs. computers, emails vs. files. Personal and Ubiquitous Computing, London, v. 22, n. 4, p. 621-632, 2018.

BRUCE, H.; JONES, W.; DUMAIS, S. Information behaviour that keeps found things found. Information Research, Sheffield, v. 10, n. 1, 2004.

BUSH, V. As we may think. The atlantic monthly, [S.l.], v. 176, n. 1, p. 101$108,1945$. 
CASE, D.; GIVEN, L. M. Looking for informantion. 4. ed. [S.l.]: Emerald, 2016.

CASE, D. O.; O'CONNOR, L. G. What's the use? Measuring the frequency of studies of information outcomes. Journal of the Association for Information Science and Technology, Hoboken, v. 67, n. 3, p. 649-661, 2016.

CHARLOT, B. A pesquisa educacional entre conhecimentos, políticas e práticas: especificidades e desafios de uma área de saber. Revista Brasileira de educação, Rio de Janeiro, v. 11, n. 31, p. 7-18, 2006.

ETZEL, B.; THOMAS, P. Personal information management. In: ETZEL, B.; THOMAS, P. Personal Information Management. London: Palgrave, 1996. p. 8-17.

FRY, J. Scholarly research and information practices: a domain analytic approach. Information processing and management, Oxford, v. 42, p. 299316, 2006.

GIVEN, L. M.; WILLSON, R. Information technology and the humanities scholar: Documenting digital research practices. JASIST, [S.l.], v. 69, n. 6, p. 807-819, 2018.

GREIFENEDER, E. Trends in information behaviour research. In: PROCEEDINGS OF ISIC, THE INFORMATION BEHAVIOUR CONFERENCE, 10., 2014, Leeds. Proceedings [...] Leeds: Jagiellonian University, 2014.

HASSAN, S. et al. Measuring social media activity of scientific literature: an exhaustive comparison of Scopus and novel altmetrics big data. Scientometrics, Dordrecht, v. 113, p. 1037-1057, 2017.

HEMMINGER, B. M. et al. Information seeking behavior of academic scientists. Journal of the American society for information science and technology, Hoboken, v. 58, n. 14, p. 2205-2225, 2007.

HJØRLAND, B. Domain analysis. Knowledge Organization, Baden Baden, v.44, n.6, p. 436-464, 2017.

HWANG, Y.; LIN, H.; SHIN, D. Knowledge system commitment and knowledge sharing intention: the role of personal information management motivation. International Journal of Information Management, Oxford, v. 39, p. 220-227, 2018. 
JAMALI, H. R.; ASADI, S. Google and the scholar: the role of Google in scientists' information-seeking behaviour. Online Information Review, Bingley, v.34, n.2, p. 282-294, 2010.

JONES, W. Personal information management. Annual review of information science and technology, Hoboken, v. 41, n.1, p. 453-504, 2007.

JONES, W. et al. Making it real: Towards practical progress in the management of personal information. In: CHI: conference extended abstracts on human factors in computing systems, 2016, [s.l.]. Proceedings [...]. [S. 1.: s. n.], [2016]. p. 571-582.

JONES, W.; TEEVAN, J. Personal information management. Seattle: University Washington Press, 2007.

JONES, W.; WENNING, A.; BRUCE, H. How do people re-find files, emails and web pages? In: ICONFERENCE 2014, [s.l.]. Proceedings [...]. [S. 1.: s. n.], 2014.

KEMMAN, M.; KLEPPE, M.; SCAGLIOLA, S. 'Just Google It'. In: Mills, C.; Pidd, M.; Ward, E. Proceedings of the Digital Humanities Congress 2012. Sheffield: HRI Online Publications, 2014. Studies in the Digital Humanities.

KING, D. W.; TENOPIR, C. Using and reading scholarly literature. In: WILLIAMS, M. E. (ed.). Annual Review of Information Science and Technology, Hoboken, v. 34, p.423-477, 2001

$\mathrm{KOH}$, K. et al. Information seeking and beyond: impacts of studying different forms of information behavior. Proceedings of the Association for Information Science and Technology, [S.l.], v. 52, n. 1, p. 1-5, 2015.

MCGEACHIN, R. B. The Impact of Electronic Bibliographic Databases and Electronic Journal Articles on the Scholar's Information-Seeking Behavior and Personal Collection of "Reprints". Science \& Technology Libraries, [S.l.], v. 25, n. 1-2, p. 127-137, 2004.

NEWMAN, M. L.; SACK, J. Information workflow of academic researchers in the evolving information environment: an interview study. Learned publishing, Hoboken, v. 26 n. 2, p. 124-131, 2013

NIU, X.; HEMMINGER, B. M. A study of factors that affect the information-seeking behavior of academic scientists. Journal of the American 
Society for Information Science and Technology, Hoboken, v. 63, n. 2, p. 336-353, 2012.

$\mathrm{OH}, \mathrm{K}$. E. The process of organizing personal information. Dissertation (Doctor in Philosophy) - State University of New Jersey, New Brunswick, 2013.

PARÉ, F. X. Personal Information Management among office support staff in a university environment: an exploratory study. 2011. $214 \mathrm{f}$. Tese (Doutorado em Filosofia - Estudos de informação) - McGill University, School of Information Studies, Montreal, 2011.

STEWART, K. N.; BASIC, J. Information encountering and management in information literacy instruction of undergraduate, students. International Journal of Information Management, Oxford, v. 34, n. 2, p. 74-79, 2014.

TALJA, S.; MAULA, H. Reasons for the use and non-use of electronic journals and databases: a domain analytic study in four scholarly disciplines. Journal of documentation, Bingley, v. 59, n. 6, p. 673-691, 2003.

TENOPIR, C. Use and users of electronic library resources: an overview and analysis of recent research studies. Washington, D.C: Council on library and Information Resources, 2003.

TENOPIR, C. et al. Scholarly article seeking, reading, and use: a continuing evolution from print to electronic in the sciences and social sciences. Learned Publishing, Hoboken, v. 28, n. 2, p. 93-105, 2015.

VECHIATO, F. L.; FARIAS, G. B. de. Serendipidade no contexto da Ciência da Informação: perspectivas para os estudos com sujeitos informacionais.

Encontros Bibli: revista eletrônica de Biblioteconomia e Ciência da Informação, [S. 1.], v. 25, p. 01-23, 2020. DOI: 10.5007/15182924.2020.e72056. Disponível em: https://periodicos.ufsc.br/index.php/eb/article/view/1518-2924.2020.e72056. Acesso em: 18 set. 2021.

WARRAICH, N. F.; ALI, I.; YASMEEN, S. Keeping found things found Challenges and usefulness of personal information management among academicians. Information and Learning Science, [S.l.], v. 119, n. 12, p. 712720, 2018.

WELLINGS, S.; CASSELDEN, B. An exploration into the information-seeking behaviours of engineers and scientists. Journal of Librarianship and Information Science, London, v. 51, n. 3, p. 789-800, 2017. 
WILSON, T. D. Human information behavior. Informing science, [S.l.], v. 3, n. 2, p. 49-56, 2000.

Financiamento: Esta pesquisa foi desenvolvida com o apoio do Conselho Nacional de Pesquisa CNPQ.

\title{
Personal information management and its impact on scientific production of researchers in the domain of education
}

\begin{abstract}
The development of information technologies has affected the way researchers deal with the information used in conducting and communicating the results of their research. The objective of this study was to identify the strategies and resources used by Brazilian researchers in the Education domain to organize their collections and to verify if the difficulties in the management of these collections affect the scientific production of these researchers. To conduct the data collection, an electronic questionnaire was applied to researchers linked to the ten Brazilian universities best placed in the Folha de São Paulo ranking in three subsequent evaluations. A total of 205 responses were obtained, 163 of which were considered valid for analysis. The data were analyzed using the Statistical Package for the Social Sciences software and the Chi-Square statistical test was applied in order to verify the association of the distribution of the participants' responses between the study variables. The results showed that the minority of participants uses specific tools to manage their collections. It was also found that difficulty in managing their collections has impacted on the scientific production of the participants and that those who have undergone some training on tools to organize and store their documents face less difficulty in recovering materials from their personal collection. It is intended that the research results can serve as a basis for the preparation of librarians to serve this group of researchers and for the adaptation and proposition of information products and services related to PIM.
\end{abstract}

Keywords: Personal Information Management; PIM; Information-seeking behavior; Information-keeping behavior; Digital humanities

Recebido: $28 / 09 / 2020$

Aceito: 20/04/2021 


\section{Declaração de autoria}

Concepção e elaboração do estudo: Helen de Castro S. Casarin, Cátia Candida Almeida.

Coleta de dados: Helen de Castro S. Casarin.

Análise e interpretação de dados: Helen de Castro S. Casarin, Cátia Candida Almeida.

Redação: Helen de Castro S. Casarin, Cátia Candida Almeida.

Revisão crítica do manuscrito: Helen de Castro S. Casarin, Cátia Candida Almeida.

\section{Como citar}

CASARIN, Helen de Castro S.; ALMEIDA, Cátia Candida. Personal information management e seu impacto na produção científica de pesquisadores do domínio da educação. Em Questão, Porto Alegre, v. 28, n. 1, p. 258-280, 2022. DOI: http://dx.doi.org/10.19132/1808-5245281.258-280

${ }^{1}$ DIEKEMA, A.R.; OLSEN, M.W. Teacher personal information management (PDIM) practices: finding, keeping, and re-finding information. Journal of the Association for Information Science and Technology, [S.1.], v. 65, n. 11, p. 2261-2277, 2014.

${ }^{2}$ Kari, J. Conceptualizing the personal outcomes of information. Information Research, Sheffield, v. 12, n. 2, 2007.

${ }^{3}$ HENDERSON, Sarah. How do people manage their documents?: an empirical investigation into personal document management practices among knowledge workers. 2009. Tese (Doutorado) - University of Auckland, Auckland, 2009.

${ }^{4}$ Mote, L. (1962). Reasons for the variations in the information needs of scientists. Journal of Documentation, [S.1.], v. 18, p. 169-175, 1962.

"5"Em domínio de alta dispersão, abrange assuntos amplos (o número de diferentes temas de pesquisa é grande) e a organização da literatura utilizada pouco clara ou de forma pouco útil à luz dos interesses e problemas de pesquisa dos pesquisadores.” (BATES, 1996, p. 156, tradução nossa). 